\title{
Motor Competence Assessment - adaptação cultural para o Brasil (MCA-BR)
}

\author{
Motor Competence Assessment - cultural adaptation for Brazil (MCA-BR) \\ Motor Competence Assessment - adaptación cultural para Brasil (MCA-BR) \\ Cristina dos Santos Cardoso de Sá', Carlos Luz², Luis Paulo Rodrigues³ ${ }^{3}$ Rita Cordovil ${ }^{4}$
}

RESUMO I Adaptou-se para o português do Brasil o Motor Competence Assessment (MCA). Dois profissionais proficientes em português do Brasil traduziram, de forma independente, o MCA para a língua portuguesa (T1 e T2). Em seguida, gerou-se a versão traduzida de consenso (TU). Dois tradutores realizaram duas retroversões para português de Portugal (RT1 e RT2) da versão TU. Um novo processo de consenso entre tradutores e pesquisadores resultou em uma versão em português de Portugal (RTfinal), que foi comparada à versão original em busca de possíveis diferenças semânticas. A versão do instrumento em português do Brasil (TU), denominada "Avaliação da Competência Motora”, foi revisada por uma comissão de especialistas composta por um fisioterapeuta e dois educadores físicos para verificação do conteúdo, o que gerou a segunda versão de concordância (Tfinal). A Tfinal foi encaminhada a um dos autores da avaliação original para verificar a pertinência da versão em português do Brasil. Após essa etapa, 20 fisioterapeutas e 20 educadores físicos verificaram a aplicabilidade da avaliação. O MCA foi, então, adaptado culturalmente para o Brasil, sendo um instrumento relevante dado que ele avalia a competência motora sem limite de idade, podendo auxiliar no acompanhamento do desenvolvimento motor de indivíduos. Descritores | Desenvolvimento Infantil; Movimento; Criança; Adolescente.

ABSTRACT | We adapted the Motor Competence Assessment (MCA) to Brazilian Portuguese. Two professionals proficient in Brazilian Portuguese independently translated the MCA into the Portuguese language ( $\mathrm{T} 1$ and $\mathrm{T} 2$ ). Then, the translated version of consensus (TU) was generated. Two translators performed two back-translations in European Portuguese (BT1 and BT2) of the TU version. A new consensus process between translators and researchers resulted in a European Portuguese version (BTfinal), which was compared to the original version in search of possible semantic differences. A committee of experts, composed of a physical therapist and two physical educators to verify the content, reviewed the version of the instrument in Brazilian Portuguese (TU), called "Avaliação da Competência Motora," which generated the second version of agreement (Tfinal). We sent the Tfinal to one of the authors of the original evaluation to verify the relevance of the Brazilian Portuguese version. After this step, 20 physical therapists and 20 physical educators verified the applicability of the evaluation model. The MCA was then culturally adapted for Brazil. It is a relevant instrument as it evaluates motor competence without age limit, and can assist in monitoring the motor development of individuals. Keywords | Child Development; Movement; Child; Adolescent.

RESUMEN I Se adaptó al portugués brasileño la Motor Competence Assessment (MCA). Dos profesionales con dominio del portugués brasileño tradujeron de forma independiente la MCA al portugués (T1 y T2). Luego, se generó una versión traducida por consenso (TU). Dos traductores realizaron dos retrotraducciones al portugués de Portugal (RT1 y RT2) de la versión TU. Un nuevo proceso de consenso entre traductores e investigadores dio como resultado una

'Departamento de Ciências do Movimento Humano, Universidade Federal de São Paulo (Unifesp) - Santos (SP), Brasil.

E-mail: cristina.sa@unifesp.br. Orcid: 0000-0002-0920-6668

Escola Superior de Educação, Instituto Politécnico de Lisboa (IPL) - Lisboa, Portugal. E-mail: carlosl@eselx.ipl.pt.

Orcid: 0000-0002-9126-5375

${ }^{3}$ Escola Superior de Desporto e Lazer de Melgaço, Instituto Politécnico de Viana do Castelo (IPVC) - Viana do Castelo, Portugal.

E-mail: Iprodrigues@esdl.ipvc.pt. Orcid:0000-0002-6804-3600

${ }^{4}$ Centro Interdisciplinar de Estudo da Performance Humana (CIPER), Faculdade de Motricidade Humana, Universidade de Lisboa -

Lisboa, Portugal. E-mail: cordovil.rita@gmail.com. Orcid: 0000-0002-4907-7186 
versión al portugués de Portugal (RTfinal), que se comparó con la versión original en busca de posibles diferencias semánticas. La versión del instrumento en portugués brasileño (TU), denominada "Evaluación de la Competencia Motora", fue revisada por un comité de especialistas compuesto por un fisioterapeuta y dos educadores físicos para verificar el contenido, lo que generó la segunda versión de acuerdo (Tfinal). La Tfinal fue enviada a uno de los autores de la evaluación original para verificar la relevancia de la versión al portugués brasileño. Luego, 20 fisioterapeutas y 20 educadores físicos verificaron la aplicabilidad de la evaluación. Así la MCA tuvo adaptación cultural para Brasil y resulta ser un instrumento relevante para evaluar la competencia motora sin límite de edad y ayudar en el seguimiento del desarrollo motor de los individuos. Palabras clave | Desarrollo Infantil; Movimiento; Niño; Adolescente.

\section{INTRODUÇÃO}

Competência motora é definida como a capacidade de um indivíduo em realizar uma variedade de habilidades ou ações motoras, sejam elas finas ou grossas ${ }^{1}$, relacionadas ao desenvolvimento das habilidades motoras fundamentais (habilidades de estabilidade, locomotoras e manipulativas) ${ }^{2}$. Essas habilidades permitem a construção do repertório motor para a aquisição de habilidades mais complexas, necessárias em jogos, esportes e outras atividades durante a infância e a fase adulta ${ }^{3}$. Desse modo, a competência motora, com base no modelo teórico de Stodden et al. ${ }^{4}$, é função chave para um estilo de vida ativo e saudável.

Os protocolos de avaliação da competência motora existentes na literatura permitem identificar possíveis atrasos motores e avaliar os efeitos de expressões motoras fornecendo informações adequadas para intervenções futuras ${ }^{5}$. Há protocolos quantitativos, qualitativos e quantitativos/qualitativos.

Dentre os protocolos quantitativos (orientados ao produto) há: BOTMP e sua versão reduzida, BOT-2 $2^{6}$; ambos avaliam componentes das habilidades motoras grossas e finas como controle manual, coordenação manual e corporal, força e agilidade na faixa dos 4 e dos 14,5 anos de idade. $\mathrm{O}$ foco é avaliar a coordenação motora, e não especificamente a competência motora. $\mathrm{O} \mathrm{KTK}{ }^{7}$ avalia habilidades locomotoras e de estabilidade na faixa etária de 5 a 14 anos, mas não as habilidades manipulativas. Stay in Step avalia as habilidades de estabilidade, manipulativa, locomotora e velocidade apenas para a faixa etária de 5 a 7 anos $^{8}$. Dentre os protocolos qualitativos (orientados ao processo), há o TGMD-1 e 2,10, que avaliam habilidades locomotoras e manipulativas na faixa etária de 3 a 10 anos, mas não habilidades de estabilidade. Em 2017, foi criada a versão TGMD- $3^{11}$ com modificações em alguns itens, na subescala locomotora e nas habilidades com bola (controle de objeto/manipulativa) - mas permanece sem avaliar as habilidades de estabilidade e mantém a faixa etária.
Por fim, os protocolos quantitativos/qualitativos: MAND ${ }^{12}$ avalia habilidades finas e grossas, na faixa etária de 3,5 a 18 anos, mas não habilidades manipulativas. Há, também, ausência de similaridade entre muitas das tarefas utilizadas com atividades ou esportes que crianças e adolescentes tenham familiaridade. M-ABC- $1^{13}$ avalia destreza manual, habilidades de estabilidade e manipulativa, nas idades de 4 a 12 anos; $\mathrm{M}-\mathrm{ABC}-2^{14}$ avalia destreza manual, habilidades de estabilidade e manipulativa nas idades de 3 a 16 anos, mas não avalia habilidades locomotora.

A partir dos protocolos existentes para avaliar a competência motora, Luz et al. ${ }^{15}$, em Portugal, desenvolveram o modelo de competência motora para crianças e adolescentes portugueses baseado numa estrutura teoricamente dividida nos domínios da estabilidade, da locomoção e da manipulação, gerando uma bateria de testes de avaliação orientados ao produto, desconsiderando a idade de desenvolvimento e de fácil execução, diferentemente de outros instrumentos.

No Brasil, temos as mesmas desvantagens avaliativas observadas por Luz et al. ${ }^{15}$ a respeito da competência motora. Por esse motivo, este estudo objetivou adequar culturalmente para a língua portuguesa do Brasil o modelo de avaliação da competência motora, o Motor Competence Assessment (MCA).

\section{METODOLOGIA}

Este estudo realizou a tradução e a adaptação cultural para língua portuguesa do Brasil do modelo de avaliação da competência motora, o MCA.O procedimento metodológico seguiu as etapas recomendadas internacionalmente: tradução, síntese da tradução, retrotradução, análise em comitê de especialistas, pré-teste e versão final ${ }^{16,17}$.

A documentação, descrevendo todas as etapas para a tradução e adaptação cultural, foi enviada para o autor do modelo de avaliação original para que fosse garantida a adequação do processo de tradução realizado. Este 
estudo foi precedido por autorização formal dos autores da versão original do MCA para tradução e validação do instrumento para o idioma português do Brasil.

A descrição do modelo de avaliação da competência motora e as instruções relacionadas a sua aplicação, presentes na versão original em português de Portugal, foram traduzidas para o português do Brasil de acordo com as recomendações internacionais. As traduções foram realizadas por dois tradutores independentes, sendo que somente um deles conhecia o objetivo do estudo, mas desconhecia o modelo de avaliação. Essas duas traduções para o português do Brasil (T1 e T2) foram fundidas em uma versão única (TU) depois do consenso entre os dois tradutores e os pesquisadores responsáveis.

Após a tradução do modelo e suas instruções, TU foi retrotraduzida (RT) para o idioma original por dois outros tradutores, que desconheciam as finalidades do estudo e seguiram as mesmas regras da tradução inicial. Essas duas versões em português de Portugal (RT1 e RT2) passaram por novo processo de consenso entre tradutores e pesquisadores, resultando numa versão em português de Portugal (RTfinal), que foi comparada com a versão original em busca de possíveis diferenças semânticas.

A versão do instrumento em português do Brasil (TU) foi revisada por uma comissão de especialistas composta por um fisioterapeuta e dois educadores físicos, todos com mais de dez anos de experiência nas suas respectivas áreas de atuação, além de conhecimento dos dois idiomas, para verificação da validade do conteúdo. Para essa revisão, a comissão comparou a versão em português do Brasil (TU), item por item, com a versão original em português de Portugal, analisando a concordância entre elas e sugerindo mudanças que pudessem aprimorar a tradução. Cada item também foi avaliado quanto à relevância na avaliação do conteúdo do instrumento, verificando sua equivalência. Depois dessa revisão, preparou-se uma segunda versão para concordância, que foi encaminhada a um dos autores do modelo de avaliação original para verificar o entendimento da versão brasileira do MCA, chegando a uma versão brasileira final (Tfinal).

A Figura 1 apresenta o fluxograma que esquematiza o processo de tradução até a obtenção da Tfinal.

Após essa etapa, a versão Tfinal foi entregue a 20 fisioterapeutas e 20 educadores físicos, com no mínimo cinco anos de experiência em suas respectivas áreas específicas, para a verificação da aplicabilidade do MCA traduzido e adaptado. Foram analisados os seguintes aspectos: interpretação em relação à tarefa a ser executada (instrução); registro da resposta de cada item avaliado pelo MCA; e obtenção da pontuação obtida em cada tarefa do MCA.

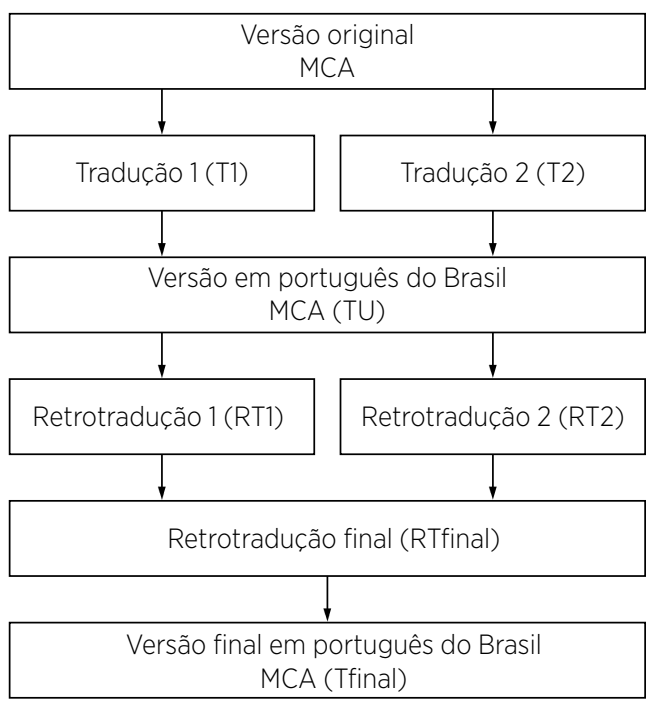

Figura 1. Fluxograma do processo de tradução e adaptação do MCA

\section{INSTRUMENTO}

O modelo MCA desenvolvido por Luz et al. ${ }^{15}$ é composto por seis tarefas quantitativas: duas de estabilidade, duas locomotoras e duas manipulativas.

As tarefas de estabilidade são: transferência lateral sobre plataformas ou transposição de plataformas $(\mathrm{TP})^{7}$, que consiste na transposição lateral de duas plataformas durante 20s; saltos laterais $(\mathrm{SL})^{7}$, que consistem em saltar lateralmente, com ambos os pés, que deverão ser mantidos juntos, durante $15 \mathrm{~s}$ - tão rapidamente quanto possível e de um lado para outro de uma viga de madeira $(60 \mathrm{~cm}$ de comprimento $\times 4 \mathrm{~cm}$ de altura $\times 2 \mathrm{~cm}$ de largura) dentro de uma área delimitada.

As tarefas locomotoras são: Shuttle Run (SHR) ${ }^{18}$, que consiste na realização de um percurso pré-determinado $(4 \times 10 \mathrm{~m})$, combinando a velocidade máxima de execução com a coordenação de pegar, transportar e colocar um pequeno bloco arredondado $(10 \mathrm{~cm}$ de altura, $5 \mathrm{~cm}$ de diâmetro) num lugar pré-determinado; e Salto com os pés unidos, ou Standing Long Jump (SLJ) ${ }^{19}$, que consiste em atingir a máxima distância num salto em comprimento com os pés unidos.

As tarefas manipulativas são: Velocidade de Lançamento $(\mathrm{VL})^{4}$, que consiste em lançar uma bola por cima do ombro, na velocidade máxima possível, contra uma parede sem correr, mas com a possibilidade de um ou dois passos para um equilíbrio preparatório. Velocidade de chutar (VP), que consiste em chutar uma bola de futebol na velocidade máxima possível, contra uma parede - chutando sem correr, mas com a possibilidade de um ou dois passos para um balanço preparatório. 
Após a realização das seis tarefas, os escores padronizados são calculados para cada tarefa. $\mathrm{Na}$ sequência, o escore para cada categoria (estabilidade, locomotora e manipulativa) é calculado pela soma da pontuação das duas tarefas representativas em cada categoria. Dada a natureza da tarefa (SHR) (em segundos), os resultados são invertidos, ou seja: realiza-se a subtração, e não a soma, das tarefas. Posteriormente, são calculados os escores padronizados por categoria, que são, na sequência, transformados em t-scores. O escore total da competência motora é calculado a partir das médias dos z-escores de todas as categorias e posteriormente transformado em z-escore; e então, por fim, em t-escores. Cabe dizer que a explicação a respeito da transformação em $t$-scores objetiva tornar os resultados mais perceptíveis para os leitores. A partir da obtenção do escore total referente à competência motora, é possível identificar e classificar a competência motora dos participantes avaliados em baixa ou alta competência motora.

\section{Participantes}

Para verificar a aplicabilidade do instrumento traduzido e adaptado, participaram do estudo 20 fisioterapeutas e 20 educadores físicos com no mínimo cinco anos de atuação nas respectivas áreas.

\section{Procedimentos de teste e análise dos dados}

Cada fisioterapeuta e educador físico convidado a testar o modelo MCA avaliou cada item do instrumento em relação a: descrição das instruções; ilustrações; descrição de obtenção do resultado/pontuação em cada tarefa executada; e descrição da pontuação total, classificando-os para os diferentes aspectos do instrumento em: adequado como está; necessidade de inclusão de alguma questão/informação; necessidade de exclusão de alguma questão/informação; necessidade de modificação de alguma questão/informação.

A partir das informações recebidas, um banco de dados foi organizado para sistematizar a revisão da versão brasileira do MCA, tomando-se o devido cuidado para que essa revisão não modificasse o conteúdo do instrumento.

\section{RESULTADOS}

\section{Tradução do MCA}

Após a tradução, o instrumento foi denominado "Avaliação da Competência Motora" - mas optou-se pela permanência do uso da sigla em inglês, MCA, utilizada no modelo original em português de Portugal, com a adição da abreviatura de Brasil, "BR”, logo em seguida: MCA-BR. Não foram encontradas discordâncias entre os itens na comparação entre a versão brasileira e a portuguesa, realizada pela comissão de especialistas.

\section{Administração da versão traduzida e adaptada do MCA}

Em relação à descrição das instruções da versão brasileira do MCA, 26 participantes (65\%) revelaram que as instruções estavam adequadas; cinco (12,5\%) sugeriram incluir algum tipo de informação na descrição e nove $(22,5 \%)$ sugeriram alguma modificação na redação das instruções.

No que se refere às ilustrações, 35 participantes (87,5\%) revelaram que as ilustrações estão adequadas e que facilitam o entendimento para realização da avaliação, e cinco (13,5\%) sugeriram incluir figuras demonstrando o momento inicial e final da tarefa. Essa sugestão foi inserida na versão brasileira.

Em relação à descrição dos resultados obtidos em cada tarefa executada, 33 participantes $(82,5 \%)$ relataram que a descrição está adequada, quatro (10\%) sugeriram a inclusão de algum tipo de informação na descrição e três $(7,5 \%)$ sugeriram modificar a redação da descrição. Assim, foi introduzido o item pontuação.

Em relação à descrição da pontuação final, 28 participantes (70\%) indicaram que a descrição da pontuação está adequada e 12 (30\%) sugeriram acrescentar informações para a obtenção do escore final.

Com base nos resultados obtidos, optou-se por modificar as informações apresentadas na descrição das instruções das tarefas, inserindo mais ilustrações com a finalidade de facilitar e complementar o entendimento da descrição das tarefas. Verificou-se, também, a necessidade de inclusão de informação extra em relação à pontuação final (Apêndice $\mathrm{A}$ ).

O Quadro 1 apresenta as adequações nas instruções, de acordo com as sugestões dadas pelos participantes.

Quadro 1. Alterações realizadas na versão em português do Brasil do MCA após teste feito por fisioterapeutas e educadores físicos

\begin{tabular}{|l|l|}
\multicolumn{1}{|c}{ Tarefa } & \multicolumn{1}{c|}{ Descrição das instruções } \\
\hline $\begin{array}{l}\text { Transposição de } \\
\text { plataforma }\end{array}$ & $\begin{array}{l}\text { - retirada a nomenclatura transferência lateral } \\
\text { (TP) }\end{array}$ \\
& $\begin{array}{l}\text { de plataforma } \\
\text { as dimensões das plataformas foram } \\
\text { especificadas: } 25 \mathrm{~cm} \text { de comprimento, } 25 \mathrm{~cm} \\
\text { de largura e } 2 \mathrm{~cm} \text { de espessura, com quatro } \\
\text { apoios de } 3,7 \mathrm{~cm} \text { de altura nos cantos } \\
\text { - mantendo uma distância mínima da outra } \\
\text { plataforma }\end{array}$ \\
\hline Saltos laterais (SL) & - ultrapassar uma viga de madeira
\end{tabular}

(continua) 
Quadro 1. Continuação

\begin{tabular}{|l|l|}
\hline \multicolumn{1}{|c|}{ Tarefa } & \multicolumn{1}{c}{ Descrição das instruções } \\
\hline Shuttle Run (SHR) & - 10 m percorrido 4 vezes \\
- pegar dois pequenos blocos arredondados & (10cm de altura, $5 \mathrm{~cm}$ de diâmetro) \\
& individualmente e transportá-los até a linha \\
& de partida/chegada
\end{tabular}

\section{DISCUSSÃO}

Este estudo adaptou para o português do Brasil o instrumento MCA para avaliar a competência motora de indivíduos.

O MCA originalmente foi elaborado e escrito em português de Portugal. Por esse motivo, a apresentação da versão adaptada do instrumento para o português do Brasil é necessária,já que, apesar de ser o mesmo idioma, há palavras com significado distinto e algumas diferenças na construção gramatical e na ortografia, o que pode gerar confusões de significado tanto para quem aplica quanto para quem recebe a aplicação ${ }^{20,21}$. Faz-se necessária a administração do modelo adaptado por um grupo de profissionais com experiência na área a fim de garantir a adaptação correta do instrumento de avaliação ${ }^{21}$. Visando a aplicação do modelo MCA à população brasileira, além da tradução, o instrumento foi testado em termos de equivalência cultural, de modo que pudesse ser compreendido e interpretado pelos avaliadores (Apêndice $A$ ).

O modelo de competência motora de Luz et al..$^{15}$, o MCA para crianças e adolescentes portugueses baseia-se em uma estrutura teoricamente dividida nos domínios: de estabilidade, de locomoção e de manipulação. Ele é uma bateria de testes de avaliação, orientado ao produto, que não considera a idade de desenvolvimento e avalia indivíduos de ambos os sexos, sendo de fácil execução. Os autores relatam que selecionar apenas testes orientados ao produto garante uma avaliação objetiva, com boa sensibilidade para discriminar o nível de competência motora ao longo das idades. Esse modelo apresenta ótimo índice geral de ajuste dos dados, sugerindo que pode ser usado para representar e avaliar a competência motora de crianças até o início da vida adulta a fim de monitorar o desenvolvimento motor. Vantagens que puderam ser observadas: uso de tarefas motoras amplamente utilizadas anteriormente em pesquisas como categorias de competência motora; a parcimônia do modelo, ao contrário de outros modelos, que utilizam diversas tarefas motoras; uso de medidas quantitativas focadas no produto final o que permite uma avaliação de desempenho mais rápida com alto nível de confiabilidade ao longo do tempo. Além disso, as medidas quantitativas são discriminadoras sensíveis entre os níveis de competência motora ao longo da infância e início da vida adulta, e são correlacionadas com avaliações qualitativas das habilidades, as quais são orientadas ao processo; o tempo de aplicação da bateria de testes é pequeno; a informação do resultado é imediata, ou seja, logo após a aplicação já é possível identificar a competência motora da criança ou adulto; não possui efeito teto da idade de desenvolvimento; proporciona a magnitude das correlações entre os fatores, $o$ que permite um escore global composto pelas competências motoras em adição aos escores por categoria.

O uso do MCA permite identificar o nível de competência motora de crianças, adolescentes e adultos, permitindo identificar ainda em quais habilidades motoras fundamentais o indivíduo pode apresentar menor desempenho. Essa informação é fundamental para traçar uma intervenção adequada a cada indivíduo, assim como possibilita acompanhar seu desenvolvimento e a melhora da competência motora, permitindo, ainda, relacionar as competências motoras e o nível de atividade física ${ }^{22-25}$.

\section{CONCLUSÃO}

O MCA foi adaptado culturalmente para o Brasil e auxiliará fisioterapeutas, educadores físicos e quaisquer profissionais que atuem em pesquisa motora, seja na educação infantil, nas escolas ou clínicas, a acompanhar o desenvolvimento motor de crianças, adolescentes e adultos; planejar intervenções direcionadas para as habilidades motoras fundamentais e acompanhar a evolução delas, de modo preciso, confiável e de fácil aplicação; permitirá a criação de políticas públicas voltadas a orientações precisas e direcionadas à prática de atividades físicas e esportivas que proporcionem um estilo de vida saudável aos indivíduos.

\section{REFERÊNCIAS}

1. Fransen J, D'Hondt E, Bourgois J, Vaeyens R, Philippaerts RM, Lenoir M. Motor competence assessment in children: convergent and discriminant validity between the BOT-2 Short Form and KTK testing batteries. Res Dev Disab. 2014;35(6):1375-83. doi: 10.1016/j.ridd.2014.03.011 
2. Gallahue DL, Ozmun JC, Goodway JD. Compreendendo o desenvolvimento motor: bebês, crianças, adolescentes e adultos. 7a ed. Porto Alegre: Editora McGraw-Hill; 2013.

3. Robinson LE, Stodden DF, Barnett LM, Lopes VP, Logan SW, Rodrigues LP, et al. Motor competence and its effect on positive developmental trajectories of health. Sports Med. 2015;45:127384. doi: 10.1007/s40279-015-0351-6

4. Stodden DF, Goodway JD, Langendorfer SJ, Roberton MA, Rudisill ME, Garcia C, et al. A developmental perspective on the role of motor skill competence in physical activity: an emergent relationship. Quest. 2008;60(2):290-306. doi: 10.1080/00336297.2008.10483582

5. Hands BP. How can we best measure fundamental movement skills? Health Sciences Conference Papers. 2002;3-5.

6. Bruininks RH, Bruininks BD, Bruininks-Oseretsky test of motor proficiency: examiner's manual. 2nd ed. Circle Pines: AGS Publishing; 2005.

7. Kiphard EJ, Schilling F. Körperkoordinationstest für Kinder: KTK Manual. Göttingen: Beltz Test; 2007.

8. Larkin D, Revie G. Stay in step: A gross motor screening test for children K-2. Sydney: University of New South Wales;1994.

9. Ulrich DA. Test of gross motor development. Austin: Pro-ED. Inc.; 1985.

10. Ulrich DA. Test of gross motor development: examiner's manual. 2nd ed. Austin: Pro-ED. Inc.; 2000

11. Webster EK, Ulrich DA. Evaluation of the psychometric properties of the test of gross motor development - third edition. J Motor Learn Dev. 2017:5(1):45-58. doi: 10.1123/jmld.2016-0003

12. McCarron L. MAND: McCarron assessment of neuromuscular development, fine and gross motor abilities. Dallas: McCarronDial Systems Inc:; 1997.

13. Henderson SE, Sugden DA. Movement assessment battery for children manual. London: The Psychological Corporation; 1992.

14. Henderson S, Sugden DA, Barnett AL. Movement assessment battery for children - second edition (Movement ABC-2): examiner's manual. London: Pearson; 2007.
15. Luz C, Rodrigues LP, Almeida G, Cordovil R. Development and validation of a model of motor competence in children and adolescents. J Sci Med Sport. 2016;19:568-72. doi: 10.1016/j. jsams.2015.07.005

16. Coster WJ, Mancini MC. Recomendações para a tradução e adaptação transcultural de instrumentos para a pesquisa e a prática em terapia ocupacional. Rev Ter Ocup. 2015;26(1):50-7. doi:10.11606/issn.2238-6149.v26i1p50-57

17. Beaton DE, Bombardier C, Guillemin F, Ferraz MB. Guidelines for the process of cross-cultural adaptation of self-report measures. Spine (Phila Pa 1976). 2000;25(24):3186-91. doi:10.1097/00007632-200012150-00014

18. AAHPER. AAHPER youth fitness test manual. Washington (DC): American Alliance for Health, Physical Education, and Recreation; 1975.

19. Ara I, Moreno LA, Leiva MT, Gutin B, Casajús JA. Adiposity, physical activity, and physical fitness among children from Aragón, Spain. Obesity. 2007;15(8):1918-24. doi:10.1038/oby.2007

20. Pedroso RS, Oliveira MS, Araujo RB, Moraes JFD. Tradução, equivalência semântica e adaptação cultural do Marijuana Expectancy Questionnaire (MEQ). Psico-USF. 2004;9(2):12936. doi: 10.1590/S1413-82712004000200003

21. Helmstadter GC. Principles of psychological measurement. New York: Appleton-Century-Crofts; 1964.

22. Stodden DF, Gao Z, Langendorfer SJ, Goodway JD. Dynamic relationships between motor skill competence and healthrelated fitness in youth. Pediatr Exerc Sci. 2014;26(3):231-41. doi: 10.1123/pes.2013-0027

23. Luz C, Rodrigues LP, De Meester A, Cordovil R. The relationship between motor competence and health-related fitness in children and adolescents. PLOS ONE. 2017;12(6):1-11. doi: 10.1371/ journal.pone.0179993

24. Luz C, Cordovil R, Almeida G, Rodrigues LP. Link between motor competence and health related fitness in children and adolescents. Sports. 2017:5(2):41. doi: 10.3390/sports5020041

25. Rodrigues LP, Luz C, Cordovil R, Bezerra P, Silva B, Camões $M$, et al. Normative values of the motor competence assessment (MCA) from 3 to 23 years of age. J Sci Med Sport. 2019;22(9):1038-43. doi: 10.1016/j.jsams.2019.05.009 


\section{APÊNDICE}

\section{Apêndice A: Versão Brasileira da Avaliação da Competência motora (MCA-BR)}

\section{INSTRUÇÕES GERAIS}

O MCA completo pode ser administrado em aproximadamente 10 a 15 minutos por participante, podendo o tempo de teste variar de acordo com a idade do participante e a experiência do examinador.

Deve ser feita a demonstração e explicação verbal de cada tarefa antes da execução. Cada participante deve experimentar cada teste antes da sua administração real. Pode ser dado feedback motivacional; no entanto, não deve ser dado feedback verbal sobre o desempenho das habilidades. Nas tarefas de lançamento / chutar, os participantes são instruídos a lançar / chutar bola o mais rápido que puderem.

Inicia-se a bateria MCA realizando as tarefas estabilizadoras, na sequência as locomotoras e por fim as manipulativas.

\section{MATERIAIS}

- 02 plataformas de madeira $(25 \mathrm{~cm}$ de comprimento $\times 25 \mathrm{~cm}$ largura $\times 2 \mathrm{~cm}$ de espessura com quatro apoios de $3,7 \mathrm{~cm}$ de altura nos cantos)

- 01 viga de madeira $(60 \mathrm{~cm}$ de comprimento $\times 4 \mathrm{~cm}$ de altura $\times 2 \mathrm{~cm}$ de largura) parafusada numa superfície de EVA retangular $(100 \mathrm{~cm}$ de comprimento $\mathrm{x} 60 \mathrm{~cm}$ de largura).

- 02 pequenos blocos arredondados $(10 \mathrm{~cm}$ de altura, $5 \mathrm{~cm}$ de diâmetro

- Fita crepe

- 01 bola de tênis (diâmetro: 6,5cm; peso: $57 \mathrm{~g}$ )

- 01 bola de beisebol (diâmetro: $7,3 \mathrm{~cm}$; peso: $142 \mathrm{~g}$ )

- 01 bola de futebol no 3 (circunferência: $62 \mathrm{~cm}$, peso: $350 \mathrm{~g}$ ).

- 01 bola de futebol $\mathrm{n}^{\circ} 4$ (circunferência de $64 \mathrm{~cm}$, peso: $360 \mathrm{~g}$ ).

- 01 bola de futebol no5 (circunferência de $68 \mathrm{~cm}$, peso: $410 \mathrm{~g}$ ).

- 01 radar de velocidade ou 01 celular com aplicativo específico para aferir a velocidade

- 01 fita métrica

- 01 cronômetro

\section{TAREFAS ESTABILIZADORAS}

\section{Tarefa 1: Transposição de plataformas (TP) ${ }^{7}$}

Nessa tarefa o participante é instruído a transferir-se lateralmente sobre a plataforma (placa) de madeira $(25 \mathrm{~cm}$ de comprimento $\times 25 \mathrm{~cm}$ largura $\times 2 \mathrm{~cm}$ de espessura com quatro apoios de $3,7 \mathrm{~cm}$ de altura nos cantos) sem colocar os pés no chão, utilizando duas plataformas que vão se transpondo lateralmente no espaço. $O$ participante posiciona-se em pé sobre uma plataforma. A outra plataforma está posicionada no chão ao seu lado (direito ou esquerdo, como o participante preferir) mantendo uma distância mínima da outra plataforma. Quando o examinador disser "já”, ele deve pegar a plataforma livre com ambas as mãos, transpô-la para o lado oposto e transferir-se para ela, repetindo essa ação por 20 segundos o mais rapidamente possível. A tarefa deverá ser executada 2 vezes, com um intervalo de 2 minutos entre as tentativas.

Pontuação: O primeiro ponto é contado quando o participante coloca a plataforma no chão (Figura 1d) e o segundo ponto quando se coloca em cima desta os dois pés (Figura 1e), e assim sucessivamente até o fim dos 20 segundos. O resultado 
final depende do número de vezes que o participante conseguir mover as plataformas e deslocar-se em cima dela durante os 20 segundos. O melhor resultado das 2 tentativas será registrado e considerado para análise (Figura 1a, 1b, 1c, 1d, 1e, 1f).
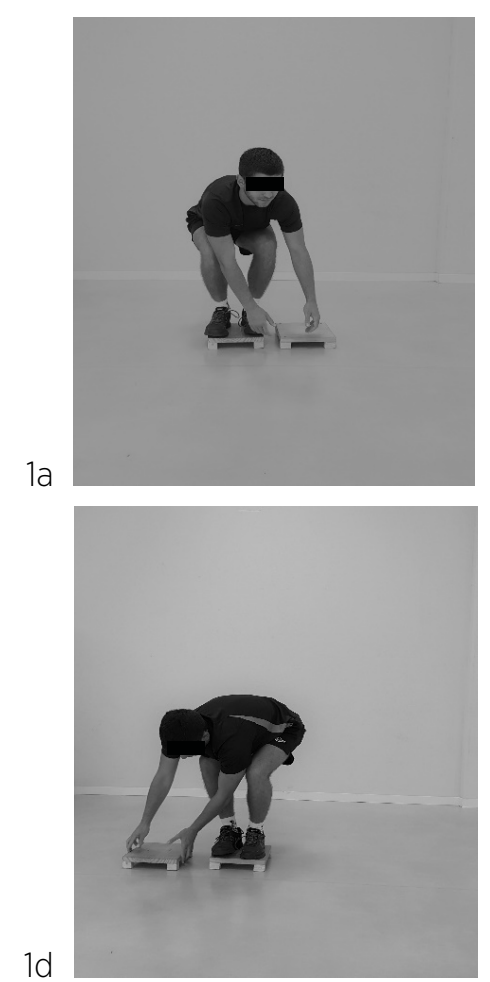
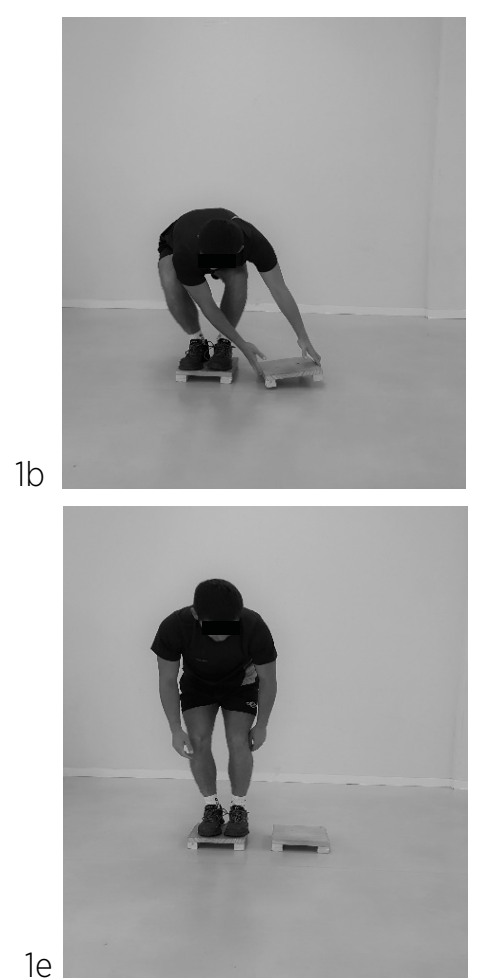

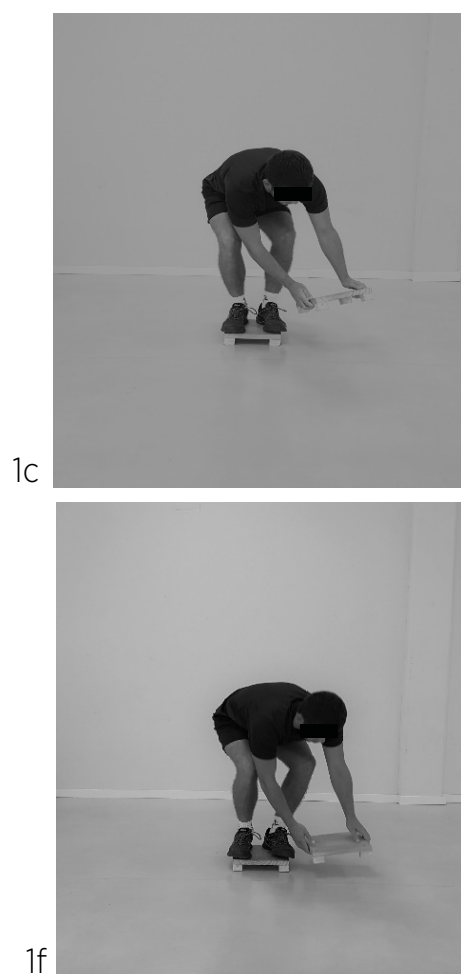

$1 f$

Figura 1. Representação da execução da tarefa transposição de plataformas (1a: posição inicial; 1b: pegar a outra plataforma; 1c e 1d) transpor a plataforma para o lado oposto; 1d: transferir-se para a plataforma; 1e: pegar novamente a plataforma)

\section{Tarefa 2: Saltos laterais $(\mathrm{SL})^{7}$}

Nessa tarefa, o participante é instruído a saltitar de um lado para o outro, com os dois pés unidos ultrapassar uma pequena viga de madeira $(60 \mathrm{~cm}$ de comprimento $\times 4 \mathrm{~cm}$ de altura $\times 2 \mathrm{~cm}$ de largura) localizada no meio de uma superfície retangular $(100 \mathrm{~cm}$ de comprimento $\times 60 \mathrm{~cm}$ de largura) o mais rápido possível por $15 \mathrm{~s}$. A tarefa deverá ser executada 2 vezes, com um intervalo de 2 minutos entre as tentativas.

Pontuação: Cada salto correto, ou seja, com os dois pés unidos sem tocar fora do retângulo e sem pisar na viga de madeira, será pontuado com 1 ponto. O resultado final resulta da soma do número de saltos nos 15 segundos de execução do teste. $\mathrm{O}$ melhor resultado será registrado e considerado para análise (Figura 2a, 2b e 2c).
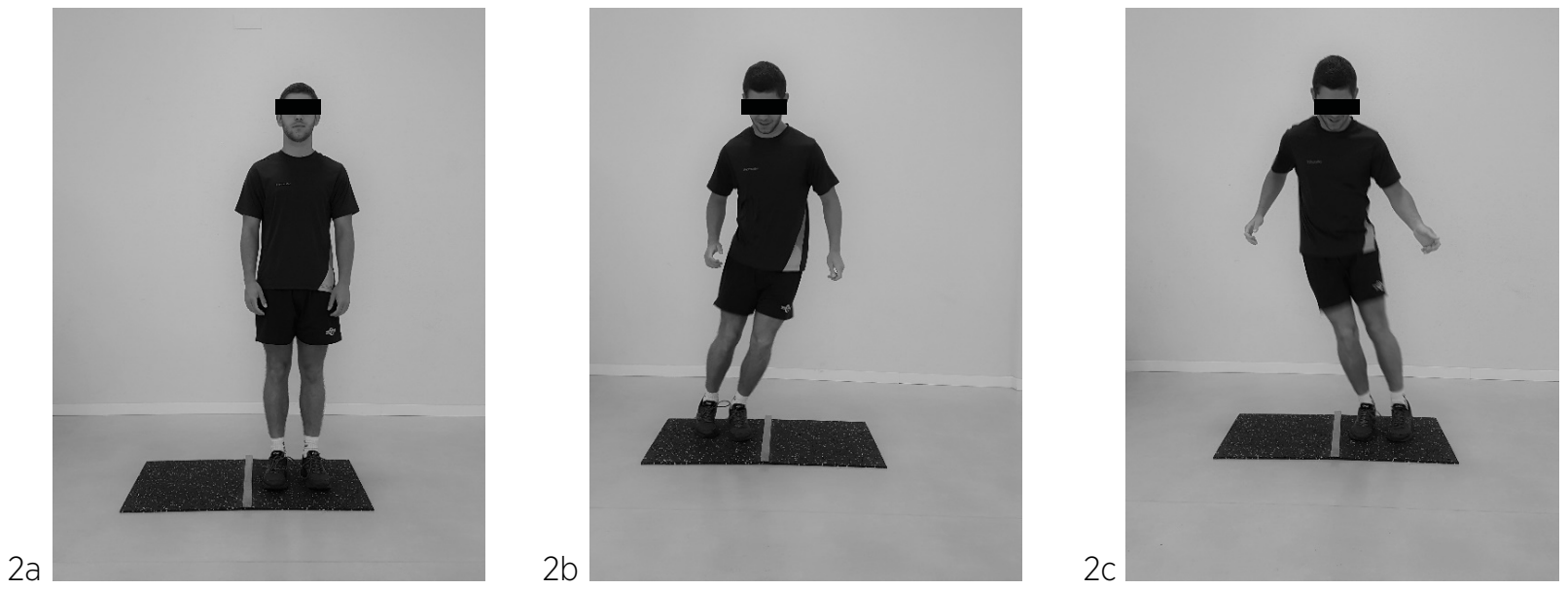

Figura 2. Representação da execução da tarefa saltos laterais (2a: posição inicial; 2b e 2c: salto lateral com os pés juntos) 


\section{TAREFAS LOCOMOTORAS}

\section{Tarefa 3: Shuttle Run (SHR) ${ }^{18}$}

A tarefa consiste na realização de um percurso pré-determinado de 10 metros percorrido 4 vezes, combinando a velocidade máxima de execução e a coordenação de pegar dois pequenos blocos arredondados $(10 \mathrm{~cm}$ de altura, $5 \mathrm{~cm}$ de diâmetro) individualmente e transportá-los até a linha de partida/chegada. O participante deve posicionar-se atrás da linha que assinala o ponto de partida, e é instruído a correr na velocidade máxima a distância de 10 metros, 4 vezes, entre a linha de partida (ponto inicial) e a linha de chegada (ponto final) sem parar. O início do teste é dado pelas palavras: “Atenção! Prepare! Já!”. Ao ouvir a palavra "Já”, o cronômetro é acionado e o participante deve correr o mais rápido possível na direção dos blocos, que estão posicionados atrás da segunda linha, deve pegar um dos blocos, distantes $25 \mathrm{~cm}$ um do outro, retornar à linha inicial (ponto de partida) e colocá-lo no chão após a linha (independentemente da posição). Depois, voltar e pegar o segundo bloco, seguindo o mesmo procedimento. O cronômetro é parado, no momento em que o participante transpõe a linha de partida/chegada com o bloco na mão, não é necessário colocar o bloco no chão. A tarefa deverá ser executada 2 vezes, com um intervalo de 2 minutos entre as tentativas.

Pontuação: o melhor tempo das 2 tentativas, ou seja, o menor tempo, será registrado e considerado para análise (Figura 3a, 3b, 3c, 3d, 3e).

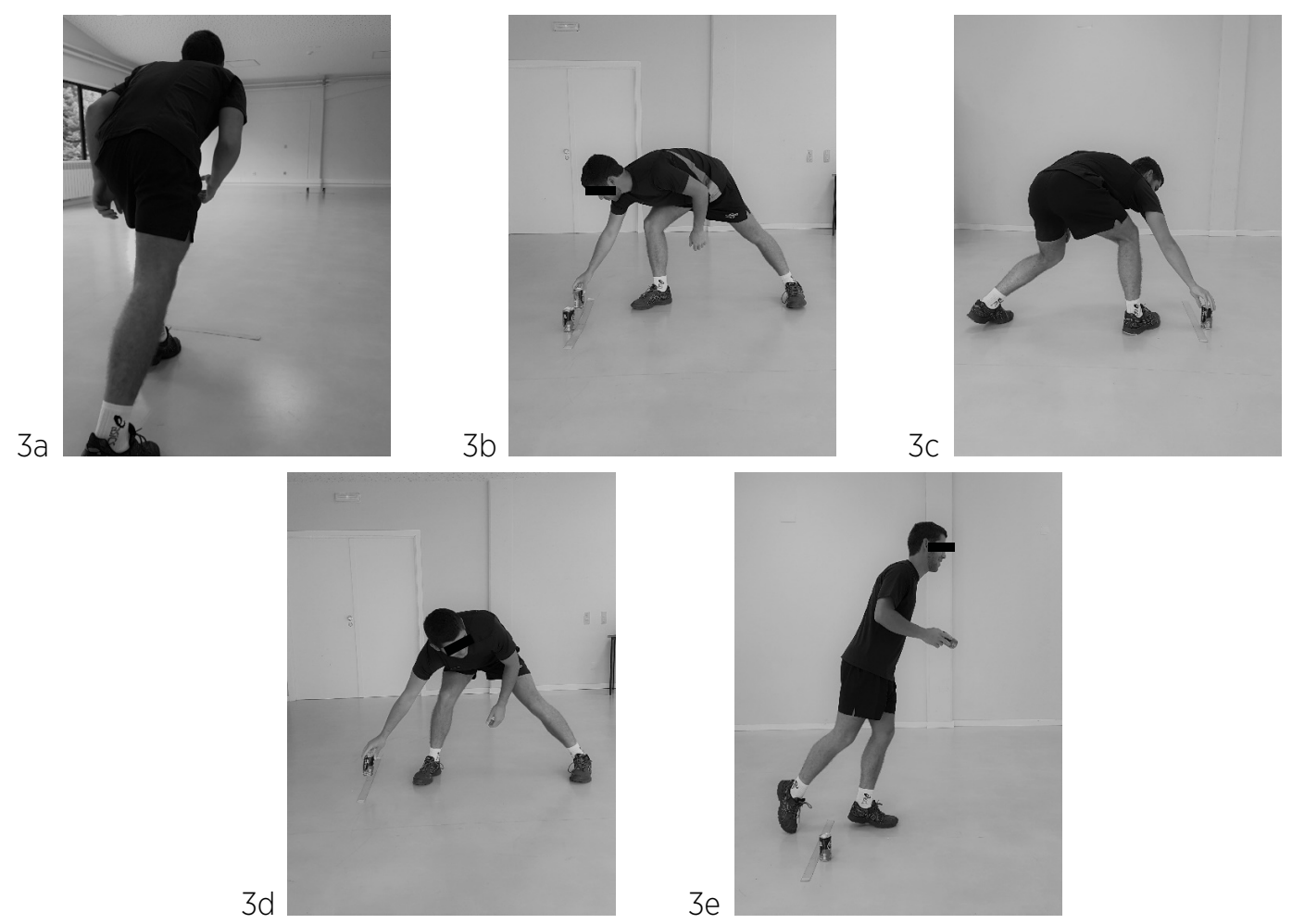

Figura 3. Representação da execução da tarefa Shuttle Run (SHR) (3a: início do percurso de 10m, linha de partida; 3b: pega o primeiro bloco na segunda linha; 3c: retorna à linha inicial e coloca o primeiro bloco; 3d: pega o segundo bloco na segunda linha; 3 e: retorna a linha de partida, com o segundo bloco).

\section{Tarefa 4: Salto com os pés unidos (Standing Long Jump - SLJ) ${ }^{19}$}

O participante é instruído a realizar o salto mais longo possível com os pés unidos, partindo da posição estática, podendo usar a ação do tronco para ganhar mais impulso. A distância alcançada será medida como a distância da linha de partida até a posição do calcanhar do pé mais próximo do ponto de partida após o salto. Esta distância será registrada em centímetros para cada salto. A tarefa deve ser executada 3 vezes.

Pontuação: A maior distância percorrida em 3 tentativas será utilizada para análise dos dados (Figura 4a , 4b, 4c e 4d). 

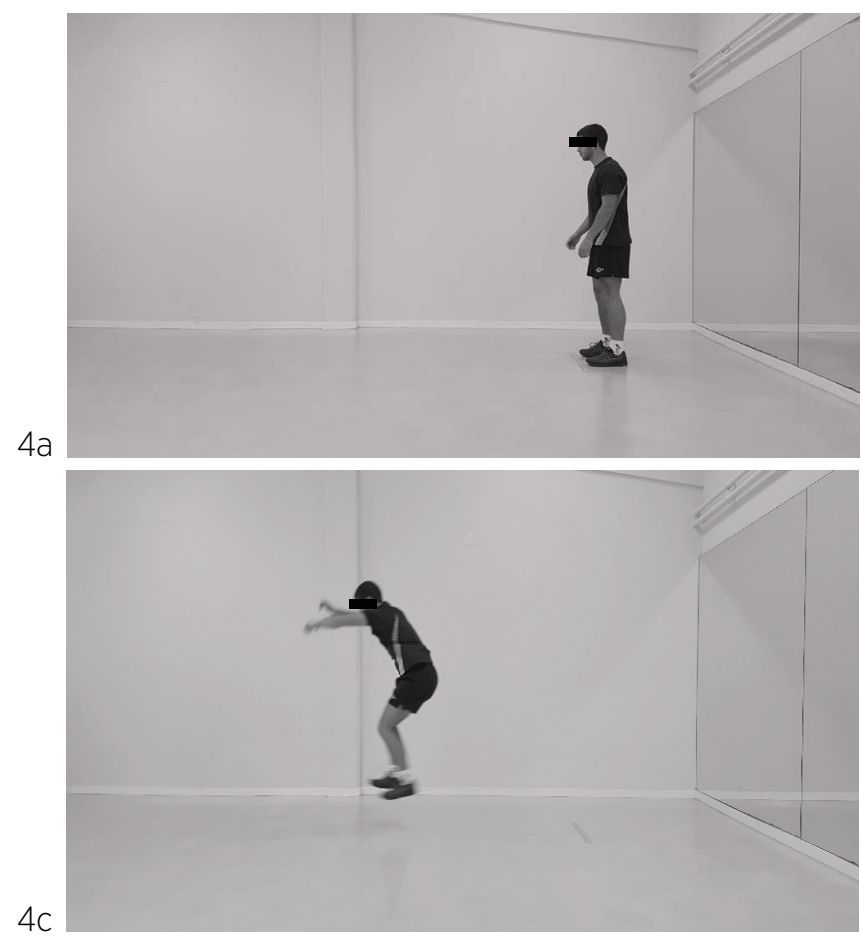

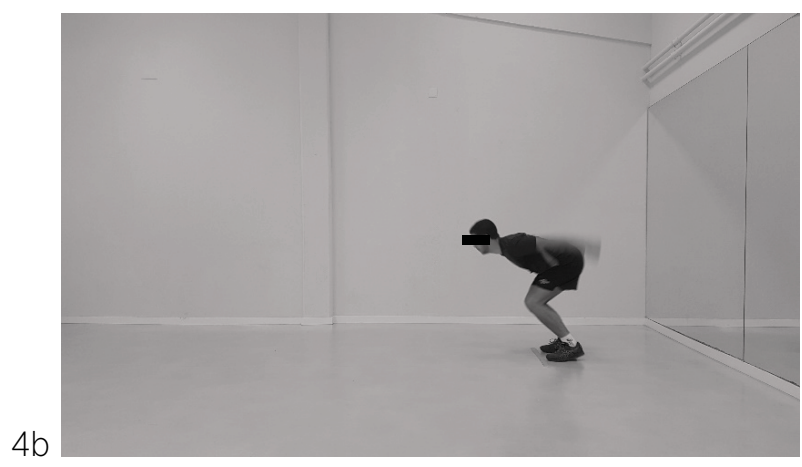

$4 \mathrm{~b}$

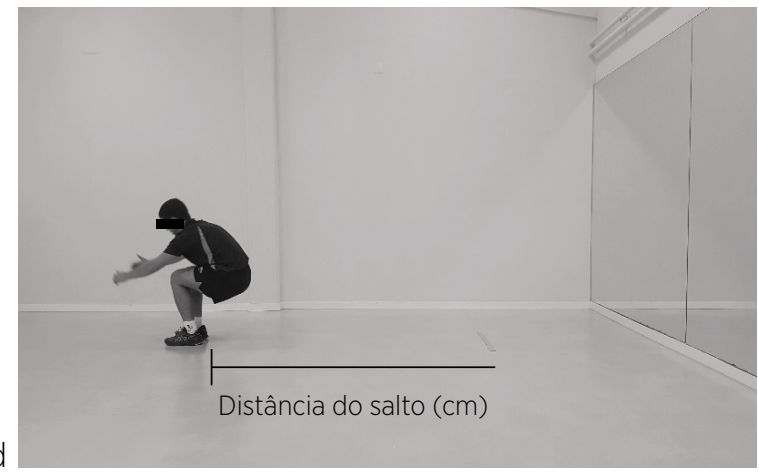

Figura 4. Representação da tarefa salto com os pés unidos (4a: posição inicial; 4b: início do salto; 4c: salto; 4d: finalização do salto)

\section{TAREFAS MANIPULATIVAS}

\section{Tarefa 5: Velocidade de lançamento ${ }^{4}$}

O participante é instruído a lançar uma bola em uma parede com máxima força A técnica de lançamento é por cima do ombro, a partir da posição em pé estática, ou seja, não há uma corrida preparatória para lançar a bola, mas há possibilidade de um equilíbrio preparatório (um ou dois passos). O participante estará em pé em uma linha demarcada a uma distância de 6 metros da parede. No meio da parede, a distância de $170 \mathrm{~cm}$ do chão, uma cruz $(40 \mathrm{~cm} \times 40 \mathrm{~cm})$ marca o alvo pretendido, (o alvo é apenas para ajudar no direcionamento da ação). Todas as tentativas feitas na direção da parede com movimento correto são contadas (lançamento por cima do ombro, e para frente, não para baixo). Para participantes entre 3 e 10 anos de idade é utilizada uma bola de tênis (diâmetro: 6,5cm; peso: $57 \mathrm{~g}$ ). Para participantes a partir dos 11 anos de idade é usada uma bola de beisebol (diâmetro: 7,3cm; peso: $142 \mathrm{~g}$ ). A velocidade de cada tentativa de arremesso será medida em m/s com um radar de velocidade (por exemplo, uma pistola de radar Pro II STALKER; ou por um aplicativo para celular, por exemplo: Speed Gun), colocado no lado da mão dominante do participante, próximo à linha no chão, acima do nível do ombro e de frente para a parede do alvo. A tarefa deve ser executada 3 vezes.

Pontuação: o maior valor de velocidade máxima das três tentativas será registrado e considerado para análise (Figura 5a e 5b).
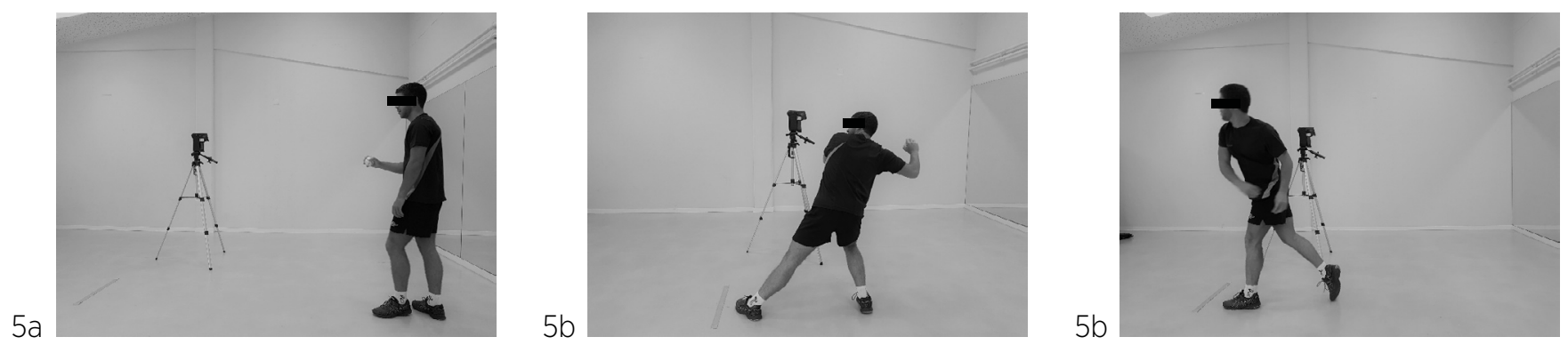

Figura 5. Representação da execução da tarefa lançamento (5a: posição inicial; 5b: início do lançamento; 5c: lançamento) 


\section{Tarefa 6: Velocidade de chute}

O participante é instruído a chutar uma bola de futebol contra uma parede com velocidade máxima a partir da posição em pé estática, ou seja, não há uma corrida preparatória para lançar a bola, mas há possibilidade de um equilíbrio preparatório (um ou dois passos). O participante estará em pé em uma linha demarcada a uma distância de 6 metros da parede. Para participantes entre 3 e 8 anos de idade utiliza-se a bola de futebol $\mathrm{n}^{\circ} 3$ (circunferência: 62 cm, peso: $350 \mathrm{~g}$ ). Para participantes de 9 e 10 anos é utilizada uma bola de futebol no4 (circunferência de $64 \mathrm{~cm}$, peso: 360g). Para participantes a partir de 10 anos de idade é utilizada uma bola de futebol nº 5 (circunferência de 6 $8 \mathrm{~cm}$, peso: $410 \mathrm{~g}$ ). O pico de velocidade da bola deve ser medido em $\mathrm{m} / \mathrm{s} \mathrm{com} \mathrm{um} \mathrm{radar} \mathrm{de} \mathrm{velocidade} \mathrm{(por} \mathrm{exemplo,}$ uma pistola radar Pro II Stalker) colocado no lado do pé dominante do participante, próximo à linha no chão a $1 \mathrm{~m}$ do chão e de frente para a parede do alvo. A tarefa será executada 3 vezes.

Pontuação: o maior valor de velocidade máxima das 3 tentativas será registrado e considerado para análise (Figura 6a, 6b, 6c).

$6 a$

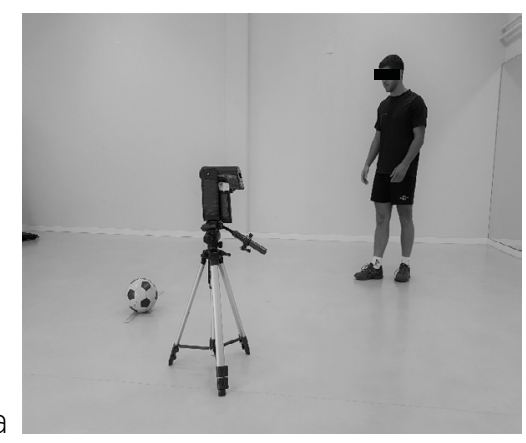

$6 b$
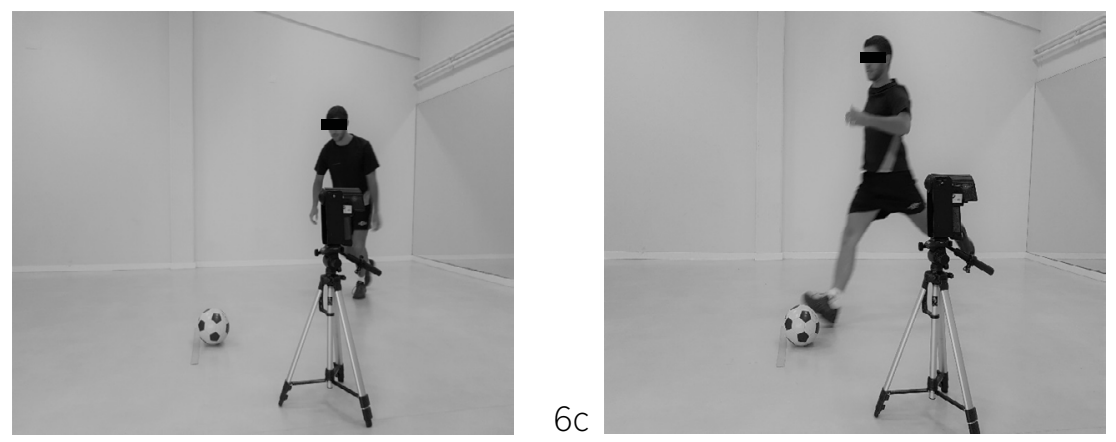

Figura 6. Representação da execução da tarefa chute (6a: posição inicial; 6b: preparação podendo dar 1 ou 2 passos; 6 c: chute)

\section{OBTENÇÃO DOS ESCORES}

Após a realização das seis tarefas, é calculado o z-escore para cada tarefa. Na sequência soma-se o z-escore das duas tarefas da mesma categoria e obtém-se o z- escore para cada categoria (estabilidade, locomotora e manipulativa). Para a categoria locomotora, como no Shuttle Run (SHR), quanto menor o tempo, melhor o resultado, o z-escore dessa tarefa será invertido. Para calcular o escore total da competência motora (escore MC) soma-se o z-escore das três categorias e calcula-se a média do z-escore. Após calcular a média, obtém-se o z-escore total, (escore MC), e converte para $\mathrm{t}$-escore, usando a fórmula $\mathrm{t}$-escore $=(\mathrm{z}$-escore $\mathrm{x} 10)+50$.

A partir da obtenção do escore MC é possível identificar e classificar a competência motora dos participantes avaliados em baixa ou alta competência motora. 